\title{
Diode Laser Assisted Excision and Low Level Laser Therapy in the Management of Mucus Extravasation Cysts: A Case Series
}

\author{
Abdul Ahad ${ }^{1 *}$, Shruti Tandon ${ }^{2}$, Arundeep Kaur Lamba ${ }^{2}$, Farrukh Faraz ${ }^{2}$, Parimal Anand ${ }^{2}$, Abdul Aleem ${ }^{1}$ \\ 'Department of Periodontics, Ziauddin Ahmad Dental College and Hospital, Aligarh Muslim University, Aligarh, India \\ ${ }^{2}$ Department of Periodontics, Maulana Azad Institute of Dental Sciences, New Delhi, India
}

\author{
*Correspondence to \\ Abdul Ahad, Department of \\ Periodontics, Ziauddin Ahmad \\ Dental College and Hospital, \\ Aligarh Muslim University, Aligarh - \\ 202002, India. \\ Email: alig.maids@gmail.com
}

Published online 27 June 2017

\begin{abstract}
Introduction: Mucus extravasation cyst is a commonly occurring lesion in oral cavity that may result from traumatic severance of a salivary gland duct with subsequent extravasation of mucus into fibrous connective tissue. After a conventional excision or marsupialization, recurrence is not uncommon. Diode laser offers an effective modality for management of such lesions.

Case Reports: Four patients were referred with painless fluctuant swellings on labial and buccal mucosa. After recording history and clinical examination, provisional diagnosis of mucocele was made. All the lesions were excised with a diode laser and biopsy was performed. Surgical wounds were treated with low-level laser therapy (LLLT).

Results: Uneventful healing was observed in all 4 cases. Significant reduction in postoperative discomfort was recorded after application of LLLT. Histopathological findings were suggestive of mucus extravasation cysts.

Conclusion: Diode laser appears to be a good alternative to conventional modalities for the management of mucus extravasation cysts.

Keywords: Mucocele; Mucus extravasation Cyst; Diode laser; Low-level laser therapy.
\end{abstract}

\section{Introduction}

Mucocele is a commonly occurring lesion in the oral cavity, containing mucin, that may result from trauma to minor salivary glands. Clinically, mucocele's size ranges from few millimeters to centimeters in diameter. It is usually painless, fluctuant, and imparts transparent to bluish hue to the lesion. Etiopathogenesis of mucocele has been well-described by Yamasoba et $\mathrm{al}^{1}$ that includes trauma and obstruction of salivary ducts of minor salivary glands. These may result in extravasation or retention of mucus. Mucus extravasation cyst is usually a dome shaped tissue swelling composed of pooled mucus that escapes into the connective tissue from a severed excretory duct. It is prevalent in second and third decades of life with no sex predilection, commonly involving lower lip, followed by tongue, buccal mucosa, palate, and retromolar area. ${ }^{2} \mathrm{Mu}-$ cus retention cyst is comparatively rare, usually found on upper labial mucosa and has peak incidence in seventh and eighth decades of life. ${ }^{3}$ Since mucocele has a high recurrence rate, various treatment modalities have been reported by clinicians, including surgical, cryosurgical, electrocautery, intralesional corticosteroids, marsupialization, micro-marsupialization, and lasers. ${ }^{4,5}$

Different kinds of lasers are available with varying wavelengths and absorption coefficient. Lasers offer advantag- es of minimal trauma, uncomplicated healing and faster recovery time when compared with conventional modalities. Diode lasers, especially that of wavelength $810 \mathrm{~nm}$, have a very high affinity for melanin and haemoglobin. This feature enables it to precisely cut, coagulate, ablate, or vaporize the target soft tissue. Additionally, diode lasers are compact, affordable and easy to move since they do not have water or air lines..$^{5-7}$

\section{Case Presentation}

Case 1

A systemically healthy 20-years-old female was referred to the department of periodontics with chief complaint of localized swelling on the left labial mucosa of lower lip since one week. There was no significant finding on extra oral examination, while intraoral examination revealed a swelling with light bluish hue on the left side of labial mucosa, about seven $\mathrm{mm}$ in diameter, soft and fluctuant on palpation (Figure 1A). It was painless, but patient was extremely worried about the condition. Provisional diagnosis of mucocele was made and marsupialization of the lesion was planned under local anaesthesia. After obtaining written informed consent, the lesion was anaesthetized with $2 \%$ lignocaine with 1:80000 adrenaline. Using a scalpel, a part of mucosa was excised, resulting 
in release of viscous yellowish liquid. After thorough irrigation with normal saline, the wound was left open for healing (Figure 1B). Ibuprofen (400 mg) was prescribed twice daily for 3 days. Patient returned after 15 days and reported reappearance and gradual increase in the size of swelling since last 3 days. On examination, a similar lesion was observed at the same site, which was soft and painful on palpation (Figure 1C). Patient was informed about the recurrent lesion and complete excision was planned with diode laser under local anaesthesia.

\section{Case 2}

A female patient aged 19 years was referred to the department of periodontics with chief complaint of localized swelling on the left labial mucosa of lower lip from last 2 months. She reported frequent disappearance and reappearance of the lesion. There was no history of pain, but the patient reported discomfort in speech and brushing. Records of her previous dental treatment at a private clinic revealed history of similar swelling in the same region which was surgically excised one year ago. There was no significant finding on extra oral examination, while intraoral examination revealed a swelling with bluish hue on the left side of labial mucosa. It was about $6 \mathrm{~mm}$ in diameter, soft and fluctuant on palpation (Figure 2A). A provisional diagnosis of mucocele was made and excision with diode laser was planned under local anaesthesia.

\section{Case 3}

A 36-year-old male was referred with complaint of a 'peasized' painless swelling on inner surface of right cheek for the last 2 weeks. He reported rapid onset and progression in size of the lesion. There was no relevant medical history. On examination, a soft, fluctuant, pink growth with a bluish hue was found on right buccal mucosa (Figure $3 \mathrm{~A}$ ). It was about eight $\mathrm{mm}$ in diameter. Oral hygiene was fair, however, several teeth were found missing with subsequent pathologic migration of remaining teeth in the first and fourth quadrant. Right mandibular second premolar was found to be carious. Provisional diagnosis of mucocele was made and excisional biopsy was planned with diode laser.

Case 4

A 30-year-old male was referred with complaint of swelling of lower lip since last one month. Rapid onset and progression of the lesion was reported. Difficulty in speech and mastication was also reported, however, there had been no incidence of pain. He also reported frequent disappearance and reappearance of the swelling. On examination, a soft fluctuant swelling of around $12 \mathrm{~mm}$ in diameter was found on left side of lower lip (Figures 4A and 4B). Provisional diagnosis of mucocele was made and excision with diode laser was planned.

\section{Management}

After routine pre-surgical blood investigations and informed consent, the lesions were removed using diode
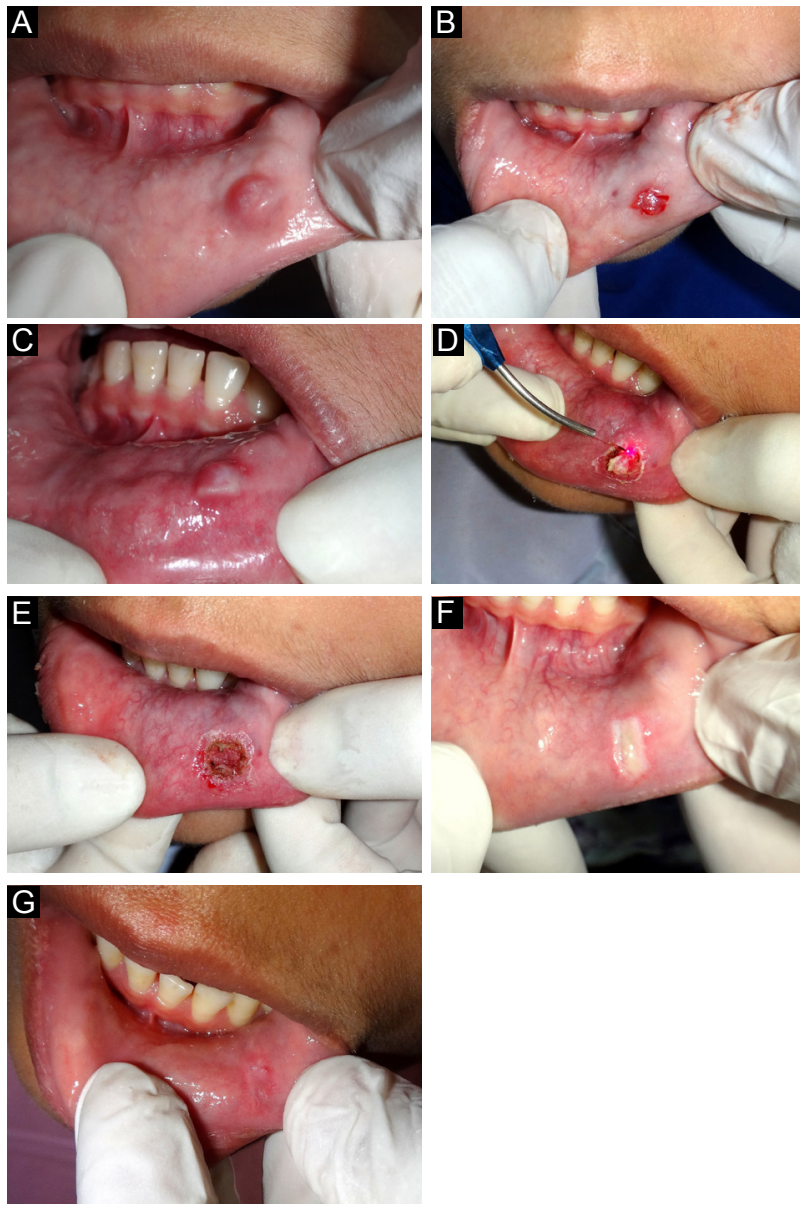

Figure 1. (A) A soft fluctuant swelling on the labial mucosa. (B) Immediate post-operative view after marsupialisation. (C) Recurrent lesion 15 days after marsupialisation. (D) Excision of the lesion by diode laser. (E) Immediate post-operative view. (F) Healing after 7 days. (G) Healing after 15 days.

laser (Picasso, AMD LASERS ${ }^{\circledR}$, Indianapolis, USA) with $810 \mathrm{~nm}$ wavelength in contact mode with pulse of 30 milliseconds duration and 30 milliseconds interval using initiated tip of $300 \mu \mathrm{m}$ diameter at a power of $2.0 \mathrm{~W}$, under local infiltration with lignocaine $2 \%$ with adrenaline 1:80 000. Surgical site was irrigated with saline during the procedure to minimize the heat. Excised lesion was put in $10 \%$ formalin and sent for biopsy. Postoperative instructions were given which included use of $0.2 \%$ chlorhexidine mouth rinse 3 times daily and topical application of aqueous based gel containing chlorhexidine for 1 week. Considering the open wound that would heal by secondary intention, LLLT was performed on first, third and seventh post-operative days to enhance the healing process. A defocused diode laser (Picasso, AMD LASERS ${ }^{\circledR}$, Indianapolis, USA) was used at one $\mathrm{mm}$ distance for two minutes. The laser used for Low-level laser therapy (LLLT) also had wavelength of $810 \mathrm{~nm}$ with power setting at $1 \mathrm{~W}$ in continuous mode.

Pain Perception

Pain perception by patient was recorded using visual an- 

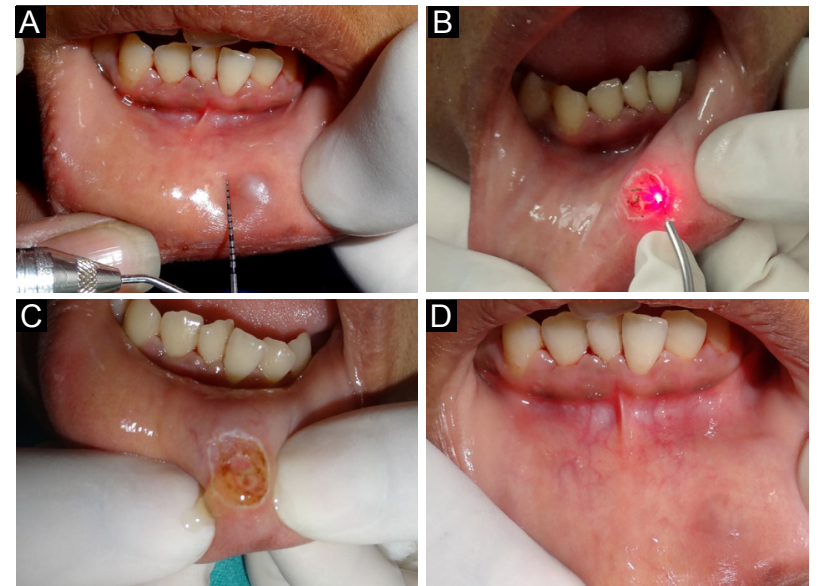

Figure 2. (A) Soft fluctuant swelling on labial mucosa. (B) Excision of the lesion with diode laser in contact mode. (C) Straw coloured liquid discharging from the lesion. (D) Complete healing after 3 weeks.
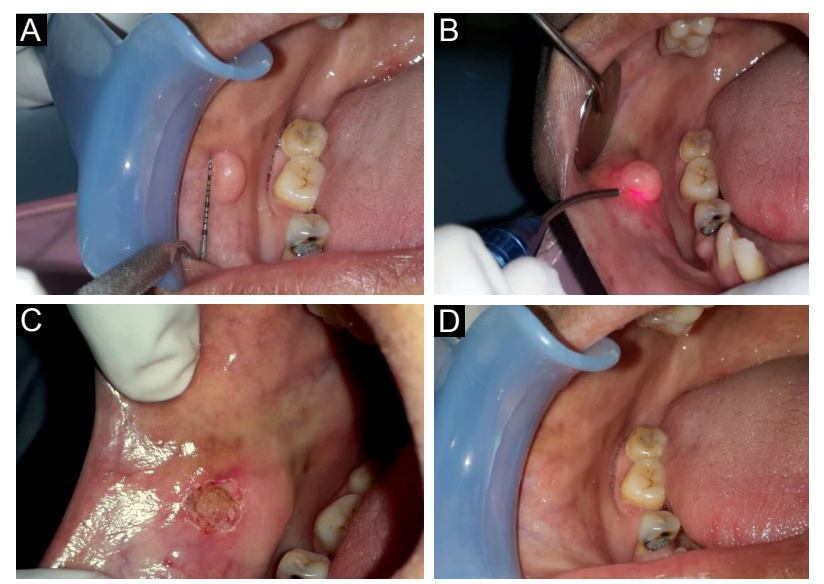

Figure 3. (A) Soft, fluctuant growth on right buccal mucosa. (B) Excision with diode laser in contact mode. (C) Immediate postoperative view. (D) Healing after 3 weeks.

alogue scale (VAS) immediately after surgery, followed by first, third and seventh postoperative day. VAS comprised a 10-cm line, with 'no pain' represented by ' 0 ' and 'unbearable pain' by '10.' Patients were instructed to mark between these 2 points. Scores between 1 and 3 were considered slight pain, scores between 3 and 6 were considered moderate pain, and scores between 6 and 10 were recorded as severe pain. Recorded values of VAS have been presented in Table 1 and Figure 5.

\section{Healing}

All cases demonstrated formation of slough on the second day after surgery. Amount of slough was significantly reduced on fourth day and completely disappeared by seventh day. Healing was uneventful in all cases. No sign of recurrence has been observed during last one year.

Histopathological Examination

All 4 cases demonstrated similar findings in histopatho-
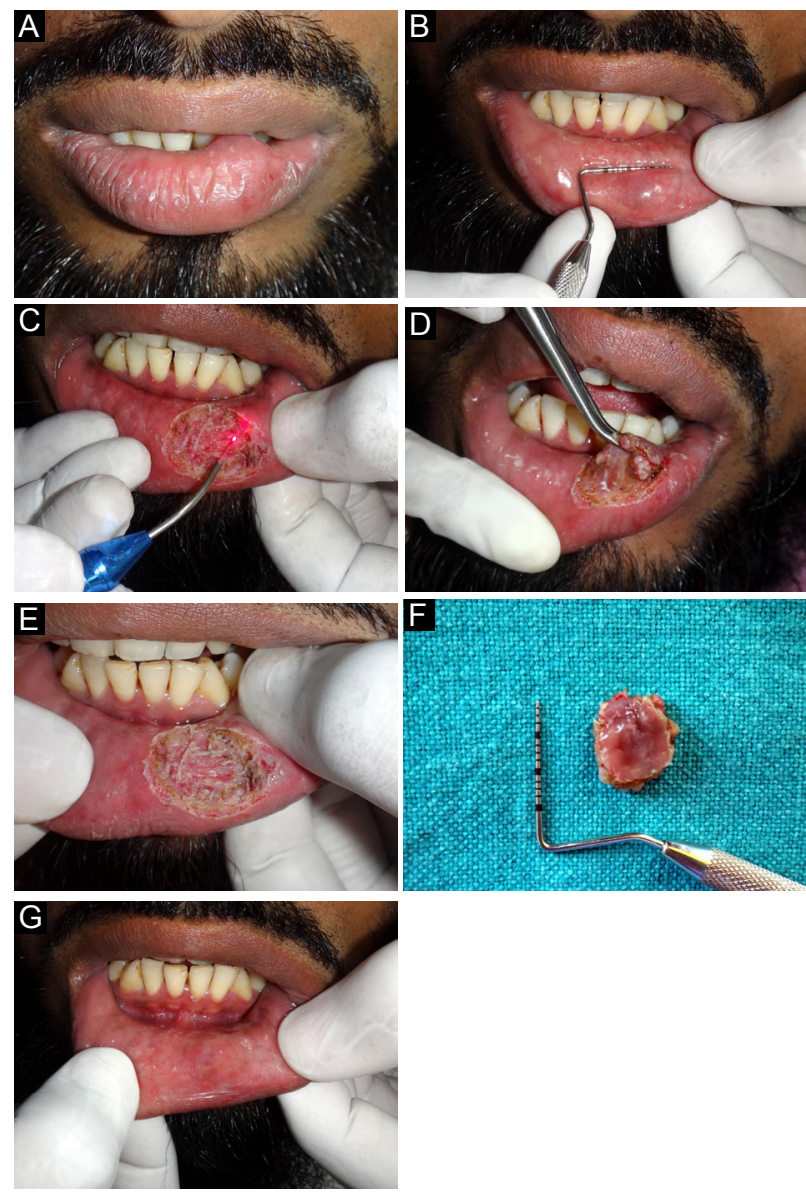

Figure 4. (A) Soft, fluctuant swelling on lower lip: extra-oral view. (B) Dimension of the lesion. (C) Excision of the lesion with diode laser. (D) Straw coloured fluid discharging from the lesion. (E) Immediate post-operative view. (F) Excised lesion. (G) Healing after 1 month.

logical sections. A cystic space was observed with a wall of compressed cellular connective tissue. Surrounding the cystic space, acute inflammatory response was present with abundance of polymorphonuclear cells, mucinophages and few eosinophils within the extravasated mucin. Normal mucous acini associated with salivary ducts were found adjacent to the field of inflammation. Abundant fibroblasts and collagen fibres were found at the periphery. These findings confirmed the diagnosis of mucus extravasation cyst.

\section{Discussion}

Mucocele is a commonly found lesion, especially in young individuals. Its incidence has been reported to be ranging from $0.4 \%$ to $0.8 \%{ }^{8}$ It is generally caused by trauma (e.g., lip biting, sharp cusps, orthodontic appliances, self-inflicted injuries) that results in the rupture of excretory duct of minor salivary glands. It usually appears as a solitary painless swelling on labial/buccal mucosa around canine-bicuspid areas, fluctuant on palpation, with a normal pink or bluish colour. Bluish coloration can be attributed to tissue cyanosis and vascular congestion associated with the stretched overlying tissue and the translucent charac- 
Table 1. Individual Scores of Pain Perception on VAS After Application of LLLT

\begin{tabular}{lccc}
\hline Patients & 1st Day & 3rd Day & 7th Day \\
\hline Patient 1 & 6 & 4 & 1 \\
Patient 2 & 5 & 4 & 0 \\
Patient 3 & 6 & 5 & 0 \\
Patient 4 & 7 & 5 & 1 \\
Mean \pm SD & $6.0 \pm 0.816$ & $4.5 \pm 0.577$ & $0.5 \pm 0.577$
\end{tabular}

Abbreviations: VAS, visual analogue scale; LLLT, Low-level laser therapy; SD, standard deviation.

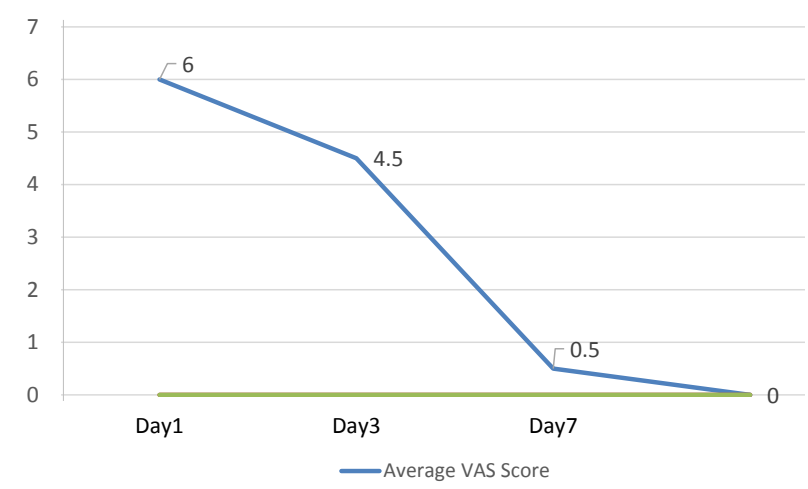

Figure 5. Changes in VAS Scores for Patient's Pain Perception Over the Period of One Week After Surgery.

ter of the accumulated fluid. ${ }^{9}$ After an appropriate differential diagnosis with other pathological forms (e.g., abscess, fibroma, lipoma, hemangioma, lymphangioma, salivary neoplasms, recurrent herpes, mucous pemphigoid, hematoma), surgical removal along with the underlying minor salivary gland represents the definitive treatment for this condition. In cases where surgical excision was not performed, particularly in the extravasation type, a cyclical increase and decrease in size of the lesion is often observed as a result of the breakage of the cyst and new production of mucin. ${ }^{10}$ Surgical excision must include the cyst, generally well-coated by a fibrous capsule, together with the minor salivary gland responsible for the mucocele, in order to avoid undesired recurrences. ${ }^{4}$

Different surgical techniques have been described in literature, depending generally on the lesion's clinical features. These include incision, complete excision, marsupialization and micro-marsupialisation. Marsupialization is a good alternative because excision or deep dissection risks vital structures such as the labial branch of the mental nerve. In traditional scalpel surgery, Baurmash proposed complete excision for small lesions and unroofing procedure for large mucoceles. ${ }^{2}$ However, following unroofing procedure, recurrence is not uncommon as we found in our first case. In that case, recurrence might have resulted from underlying glands, as it was merely unroofed instead of complete excision. Sagari et al reported micro-marsupialization as a treatment option instead of surgical excision in children. This has certain advantages such as no need of infiltrative anaesthesia, shorter surgical time and shorter post-operative healing period. Additionally, the patient compliance is also better. ${ }^{11}$ However, clinical diagnosis cannot be confirmed through histopathology in this technique. ${ }^{4}$

Cryosurgery, although widely used in the past, has the disadvantage of postoperative morbidity like oedema, irritation and delayed healing. Vaporization with argon and Nd:YAG (neodymium-doped yttrium aluminium garnet) lasers offered good alternative but biopsy sample could not be obtained while $\mathrm{CO}_{2}$ laser often results in scarring of the operated site. ${ }^{12}$

\section{Advantages of Diode Laser for Excisional Biopsy}

A diode laser was used in these cases as it offers the advantage of providing bloodless field, rapid healing and is easy to operate. ${ }^{7}$ Light of wavelength of $810 \mathrm{~nm}$ is intensely absorbed by oxygenated haemoglobin, resulting in elevation of temperature at operative site, promoting coagulation and carbonization of soft tissues, with minimum discomfort and scarring. Stimulation of clotting factor VII by diode laser resulting in enhanced haemostasis has also been reported in literature. ${ }^{13,14}$ Bactericidal effect of the diode laser has been reported by Moritz et al providing significant reduction in bacteria on the wound bed which also reduces the risk of bacteremia. ${ }^{15}$ Another important advantage is that it causes no alteration in microanatomy of biopsy specimen. ${ }^{16}$ Excessive heat may be prevented by intermittent irrigation with normal saline. Diode laser with an effective penetration depth of $2 \mathrm{~mm}$ in the tissue seal the small lymphatic vessels, thus minimizing the postoperative oedema. ${ }^{17}$ No sutures or surgical packs were given in our cases, as laser has been found to enhance wound healing by formation of a denatured protein coagulum termed as "eschar" or "biological bandage." This layer of protein coagulum protects the wound from bacteria and friction from masticatory forces. ${ }^{18}$ As seen in our cases, usually no scar formation occurs at the operated site. Similar observations have been made by Kalakonda et $\mathrm{al}^{19}$ and Zeinoun et $\mathrm{al}^{20}$ who reported that laser treated wounds exhibit less scar formation due to a fewer number of myofibroblasts, resulting in minimal wound contraction.

\section{Role of Low Level Laser Therapy in Healing of Surgical Wounds}

LLLT has been found to offer analgesic effect by stimulation of body's natural painkillers, $\beta$-endorphins and by decreasing the activity of $\mathrm{C}$ fibres. ${ }^{21}$ Healing of surgical wound has been found to be promoted by LLLT in many studies. ${ }^{21-23}$ Various mechanisms have been proposed to explain this effect of LLLT. ${ }^{22}$ At the cellular level, low power laser causes photochemical, photophysical and/or photobiological effects in the cells, leading to stimulation of certain cellular functions, particularly increased ATP production in the mitochondria. These metabolic changes inside the cells have been found to result in accelerated cell division, especially of fibroblasts, epithelial and endothelial cells, rapid production of extracellular matrix and 
movement of leucocytes, fibroblasts and epithelial cells. ${ }^{22}$ It has also been found to reduce the amount of slough formation on the wound by increased activity of macrophages and mast cells. ${ }^{21}$ The anti-inflammatory and anti-oedema effects of laser occur through acceleration of microcirculation, leading to changes in capillary hydrostatic pressure with oedema reabsorption and disposal of intermediary metabolites. ${ }^{22}$ VAS recorded in present cases showed that perceived pain was highest at the first post-surgical day and decreased to pre-surgical levels after 1 week. This is in accordance with healing patterns of mucosal wounds as demonstrated by Kalakonda et $\mathrm{al}^{19}$ and Chawla et $\mathrm{al}^{23}$ who performed LLLT after vestibuloplasty procedure and after gingival depigmentation respectively. Since present case series did not have any controls, any conclusion about role of LLLT in perceived pain cannot be drawn. However, few controlled studies have strongly suggested a clear trend in reduction of inflammation at laser treated sites. ${ }^{24}$

\section{Conclusion}

The findings in this case series of mucus extravasation cysts treated with diode laser suggest a good healing outcome, without any recurrence or alteration of microstructure of biopsy specimens. Although it was not a controlled clinical trial, patients' perception about post-operative discomfort strengthens the existing evidence that LLLT promotes wound healing on labial and buccal mucosa. Considering the advantages over other treatment modalities, diode laser appears to be a viable and safe option for management of such cases.

\section{Conflict of interests}

The authors have no conflict of interest to declare.

\section{Ethical Considerations}

Informed consent was obtained from each patient before starting the treatment procedure.

\section{References}

1. Yamasoba T, Tayama N, Syoji M, etal. Clinicostatistical study of lower lip mucoceles. Head Neck. 1990;12:316-320.

2. Baurmash HD. Mucoceles and ranulas. J Oral Maxillofac Surg. 2003;61:369-378.

3. Mustapha IZ, Boucree SA Jr. Mucocele of the upper lip: Case report of an uncommon presentation and its differential diagnosis. J Can Dent Assoc. 2004;70:318-321.

4. Chawla K, Lamba AK, Faraz F, et al. Treatment of lower lip mucocele with Er,Cr:YSGG laser: a case report. J Oral Laser Appl. 2010;10:181-185.

5. Pandey R, Pathakota KR, Koppolu P, et al. Treatment of mucocele with diode laser. J Dent Lasers. 2013;7:43-46.

6. Derikvand N, Chinipardaz Z, Ghasemi S, et al. The versatility of $980 \mathrm{~nm}$ diode laser in dentistry: a case series. J Lasers Med Sci. 2016;7(3):205-208. doi:10.15171/ jlms.2016.36.

7. Ahad A, Tandon S, Lamba AK, et al. Minimally invasive management of pericoronal abscess using $810 \mathrm{~nm}$ GaAlAs diode laser. Int J Laser Dent. 2014;4:79-82.

8. Knapp MJ. Oral Disease in 181,338 consecutive oral examination. J Am Dent Assoc. 1971;83:1288-1293.

9. Re Cecconi D, Achilli A, Tarozzi M, et al. Mucoceles of the oral cavity: a large case series (1994-2008) and a literature review. Med Oral Patol Oral Cir Bucal. 2010;15:551-556.

10. Romeo U, Palaia G, Tenore G, et al. Excision of oral mucocele by different wavelength lasers. Indian J Dent Res. 2013;24:211-215.

11. Sagari SK, Vamsi KC, Shah D, et al. Micro-marsupialization: A minimally invasive technique for mucocele in children and adolescents. J Indian Soc Pedod Prev Dent. 2012;30:188191.

12. Yagüe-García J, España-Tost AJ, Berini-Aytés L, et al. Treatment of oral mucocele - scalpel versus $\mathrm{CO}_{2}$ laser. Med Oral Patol Oral Cir Bucal. 2009;14(9):e469-e474.

13. Coleton S. Lasers in surgical periodontics and oral medicine. Dent Clin North Am. 2004;48:937-962

14. Goharkhay K, Moritz A, Wilder-Smith P, et al. Effects on oral soft tissue produced by a diode laser in vitro. Lasers Surg Med. 1999;25:401-406.

15. Moritz A, Schoop U, Goharkhay K, et al. Treatment of periodontal pockets with a diode laser. Lasers Surg Med. 1998;22:302-311.

16. Chawla K, Lamba AK, Faraz F, et al. Diode laser for excisional biopsy of peripheral ossifying fibroma. Dent Res J. 2014;11:525-30.

17. Pirnat S. Versatility of an $810 \mathrm{~nm}$ diode laser in dentistry: an overview. J Laser Health Acad. 2007;4:1-9.

18. Parker S. Lasers and soft tissue: 'fixed' soft tissue surgery. $\mathrm{Br}$ Dent J. 2007;202:247-253. doi:10.1038/bdj.2007.172.

19. Kalakonda B, Farista S, Koppolu P, et al. Evaluation of patient perceptions after vestibuloplasty procedure: a comparison of diode laser and scalpel techniques. J Clin Diagn Res. 2016;10:96-100. doi:10.7860/JCDR/2016/17623.7820.

20. Zeinoun $\mathrm{T}$, Nammour S, Dourov $\mathrm{N}$, et al. Myofibroblasts in healing laser excision wounds. Lasers Surg Med. 2001;28(1):74-79. doi:10.1002/10969101(2001)28:1<74::AID-LSM1019>3.0.CO;2-B.

21. Walsh LJ. The current status of low level laser therapy in dentistry. Part 1. Soft tissue applications. Aust Dent J. 1997;42:247-54.

22. Lins RDAU, Dantas EM, Lucena KCR, et al. Biostimulation effects of low-power laser in the repair process. An Bras Dermatol. 2010; 85:849-55.

23. Chawla K, Lamba AK, Tandon S, et al. Effect of low-level laser therapy on wound healing after depigmentation procedure: A clinical study. J Indian Soc Periodontol. 2016;20:184-8.

24. Alipanah Y, Asnaashari M, Anbari F. The effect of low level laser (GaAlAs) therapy on the post-surgical healing of full thickness wounds in rabbits. Med Laser Appl. 2011;26(3):133-138. doi:10.1016/j.mla.2011.05.005. 